\title{
ASYMPTOTIC SPECTRAL ANALYSIS OF GROWING GRAPHS: ODD GRAPHS AND SPIDERNETS
}

\author{
DAISUKE IGARASHI \\ Graduate School of Information Sciences \\ Tohoku University \\ Sendai 980-8579, Japan \\ NOBUAKI OBATA \\ Graduate School of Information Sciences \\ Tohoku University \\ Sendai 980-8579, Japan \\ E-mail: obata@math.is.tohoku.ac.jp
}

\begin{abstract}
Two new examples are given for illustrating the method of quantum decomposition in the asymptotic spectral analysis for a growing family of graphs. The odd graphs form a growing family of distance-regular graphs and the two-sided Rayleigh distribution appears in the limit of vacuum spectral distribution of the adjacency matrix. For a spidernet as well as for a growing family of spidernets the vacuum distribution of the adjacency matrix is the free Meixner law. These distributions are calculated through the Jacobi parameters obtained from structural data of graphs.
\end{abstract}

1. Introduction. Let $\mathcal{G}=(V, E)$ be a graph, where $V$ is a non-empty set and $E$ a subset of $\{\{x, y\} ; x, y \in V, x \neq y\}$. An element of $V$ is called a vertex and an element of $E$ is called an edge. Two vertices $x, y \in V$ are called adjacent if $\{x, y\} \in E$, and in that case we write $x \sim y$. Throughout this paper a graph is always assumed to be locally finite and connected. The adjacency matrix $A=\left(A_{x y}\right)_{x, y \in V}$ is defined by

$$
A_{x y}= \begin{cases}1, & \text { if } x \sim y \\ 0, & \text { otherwise }\end{cases}
$$

2000 Mathematics Subject Classification: Primary 46L53; Secondary 05C50, 60F05, 81S25.

Key words and phrases: adjacency matrix, spectral distribution, quantum decomposition, Rayleigh distribution, free Meixner law, odd graph, spidernet, tree.

Research supported by Grant-in-Aid for Scientific Research from JSPS No. 15340039.

The paper is in final form and no version of it will be published elsewhere. 
Then $A$ acts in the Hilbert space $\ell^{2}(V)$ in a natural manner as

$$
A \delta_{x}=\sum_{y \sim x} \delta_{y}, \quad x \in V,
$$

where $\left\{\delta_{x} ; x \in V\right\}$ is the canonical orthonormal basis of $\ell^{2}(V)$. We are interested in a Borel probability measure $\mu$ on $\mathbb{R}=(-\infty,+\infty)$ such that

$$
\left\langle A^{m}\right\rangle \equiv\left\langle\delta_{o}, A^{m} \delta_{o}\right\rangle=\int_{-\infty}^{+\infty} x^{m} \mu(d x), \quad m=1,2, \ldots,
$$

where $o \in V$ is a fixed origin of the graph. Note that $\left\langle\delta_{o}, A^{m} \delta_{o}\right\rangle$ is nothing but the number of $m$-step walks from $o$ to itself. We are also interested in the case where (1.1) holds in an "asymptotic" sense. This question arises from analysis of a large graph or of a growing family of graphs $\mathcal{G}_{\nu}=\left(V^{(\nu)}, E^{(\nu)}\right)$. The problem is to find a Borel probability measure $\mu$ on $\mathbb{R}$ such that

$$
\lim _{\nu \rightarrow \infty}\left\langle\left(\frac{A_{\nu}}{Z_{\nu}}\right)^{m}\right\rangle=\int_{-\infty}^{+\infty} x^{m} \mu(d x), \quad m=1,2, \ldots,
$$

where $Z_{\nu}>0$ is a normalizing constant.

The theory of interacting Fock space (see e.g., Accardi-Bożejko [2] and references cited therein) and the quantum probabilistic formulation of asymptotic spectral analysis on a large graph due to Hora $[13,14]$ motivated us to propose a new approach based on the quantum decomposition and quantum central limit theorem. The idea was first applied to discrete groups by Hashimoto [9] and to Hamming graphs by Hashimoto-ObataTabei [11]. During the recent years this new method has been systematized (see e.g., Hashimoto-Hora-Obata [10], Hora-Obata [17]) and applied to Johnson graphs, Cayley graphs of Coxeter groups, and another regular graphs, see Hora [15, 16] and Hora-Obata [18], where deformed vacuum states are also studied. More recently various concepts of independence in quantum probability theory are found to be related to certain structure of graphs, as comb graphs (Accardi-Ben Ghorbal-Obata [1]) and star graphs (Obata [22]) illustrate our viewpoint.

The main purpose of this paper is to provide two new examples. The odd graphs $O_{k}$ form a growing family of distance-regular graphs and the probability distribution $\mu$ in question (1.2) is computed explicitly, which may be called the two-sided Rayleigh distribution (Theorems 5.3 and 6.1). This limit measure has not been discussed so far in quantum probability theory, though the odd graphs have attracted attention for some combinatorial interests in algebraic graph theory (Biggs [3, 4]) and for their spectral properties (Huang-Liu [20]). The second example is a spidernet which is, strictly speaking, not regular but is highly symmetric. The probability distribution $\mu$ in question (1.1) as well as in (1.2) is shown to be a free Meixner law (Theorems 7.3 and 8.2). The free Meixner laws form a natural family of one-parameter deformations of the Kesten measures and are related with harmonic analysis on free groups (Cohen-Trenholme [8]), infinite divisible laws with respect to the free convolution (Saitoh-Yoshida [24]), and the free Lévy processes (Bożejko-Bryc [5]).

The method of quantum decomposition only requires simple structural data of a graph and allows us to avoid a heavy combinatorial argument often necessary to obtain full 
description of spectrum of the adjacency matrix. We hope that our approach is justified with explicit evidence illustrated by the two examples in this paper together with several ones discussed in the previous papers mentioned above. A self-contained introduction to the method of quantum decomposition will be available in the forthcoming monograph by Hora-Obata [19].

Acknowledgements. The authors are grateful to Professors M. Bożejko, A. Hora and H. Yoshida for helpful comments and references.

2. Quantum decomposition of the adjacency matrix. Let $\mathcal{G}=(V, E)$ be a graph and $A$ the adjacency matrix. We always fix an origin $o \in V$. Then the stratification (distance partition) of the graph is introduced:

$$
V=\bigcup_{n=0}^{\infty} V_{n}, \quad V_{n}=\{x \in V ; \partial(o, x)=n\},
$$

where $\partial$ stands for the natural distance function. For $\epsilon \in\{+,-, \circ\}$ we define $A^{\epsilon}$ by

$$
\left(A^{\epsilon}\right)_{x y}= \begin{cases}1, & \text { if } x \sim y \text { and } \partial(o, x)-\partial(o, y)=\epsilon, \\ 0, & \text { otherwise, }\end{cases}
$$

where $\epsilon$ is assigned the numbers $+1,-1,0$ according as $\epsilon=+,-, \circ$. Then the adjacency matrix $A$ is decomposed into three parts:

$$
A=A^{+}+A^{-}+A^{\circ} .
$$

We call (2.2) the quantum decomposition of $A$ and $A^{\epsilon}$ the quantum components. It is shown that $A^{+}$and $A^{-}$are mutually adjoint and $A^{\circ}$ is selfadjoint (equipped with natural domains in $\left.\ell^{2}(V)\right)$.

For each $n=0,1, \ldots$ we define a unit vector in $\ell^{2}(V)$ by

$$
\Phi_{n}=\left|V_{n}\right|^{-1 / 2} \sum_{x \in V_{n}} \delta_{x},
$$

which is called the $n$-th number vector. In particular, $\Phi_{0}=\delta_{o}$ is called the vacuum vector. Let $\Gamma(\mathcal{G})$ denote the closed subspace spanned by $\left\{\Phi_{0}, \Phi_{1}, \ldots\right\}$. Note that $\Gamma(\mathcal{G})$ is not necessarily invariant under the quantum components $A^{\epsilon}$. In this paper we shall concentrate on the case where $\Gamma(\mathcal{G})$ is invariant under the quantum components $A^{\epsilon}$, $\epsilon \in\{+,-, \circ\}$. The case where $\Gamma(\mathcal{G})$ is "asymptotically" invariant is also interesting, see e.g., Hashimoto-Hora-Obata [10], Hora-Obata [17, 18]. Here we recall the following

Proposition 2.1. Notations and assumptions being as above, assume that $\Gamma(\mathcal{G})$ is invariant under the quantum components $A^{\epsilon}, \epsilon \in\{+,-, \circ\}$. Then there exists a pair of sequences $\left(\left\{\omega_{n}\right\},\left\{\alpha_{n}\right\}\right)$ such that

$$
\begin{aligned}
& A^{+} \Phi_{n}=\sqrt{\omega_{n+1}} \Phi_{n+1}, \quad n=0,1,2, \ldots, \\
& A^{-} \Phi_{0}=0, \quad A^{-} \Phi_{n}=\sqrt{\omega_{n}} \Phi_{n-1}, \quad n=1,2, \ldots, \\
& A^{\circ} \Phi_{n}=\alpha_{n+1} \Phi_{n}, \quad n=0,1,2, \ldots .
\end{aligned}
$$

In particular, $\left(\Gamma(\mathcal{G}), A^{+}, A^{-}\right)$is an interacting Fock space associated with a Jacobi parameter $\left\{\omega_{n}\right\}$. 
REMARK 2.2. If $\mathcal{G}$ is a finite graph, the stratification (2.1) terminates at a finite $n$, the Hilbert space $\Gamma(\mathcal{G})$ is of finite dimension, and both $\left\{\omega_{n}\right\}$ and $\left\{\alpha_{n}\right\}$ are finite sequences with $\omega_{n}>0$. If $\mathcal{G}$ is an infinite graph, then $V_{n} \neq \emptyset$ for all $n$, the Hilbert space $\Gamma(\mathcal{G})$ is of infinite dimension, and both $\left\{\omega_{n}\right\}$ and $\left\{\alpha_{n}\right\}$ are infinite sequences with $\omega_{n}>0$.

The question (1.1) is equivalent to finding a Borel probability measure $\mu$ on $\mathbb{R}$ such that

$$
\int_{-\infty}^{+\infty} x^{m} \mu(d x)=\left\langle\Phi_{0},\left(A^{+}+A^{-}+A^{\circ}\right)^{m} \Phi_{0}\right\rangle, \quad m=1,2, \ldots
$$

The interacting Fock space structure allows us to use non-commutativity of the quantum components. Although not explicitly written in the literature, a homogeneous tree is the prototype of our consideration, see Remarks 7.4 and 8.3.

3. Quantum central limit theorem for distance-regular graphs. A graph $\mathcal{G}=$ $(V, E)$ is called distance-regular if given $i, j, k=0,1,2, \ldots$ the intersection number

$$
p_{i j}^{k}=|\{z \in V ; \partial(x, z)=i, \partial(y, z)=j\}|
$$

is determined independently of the choice of $x, y \in V$ satisfying $\partial(x, y)=k$.

THEOREM 3.1. Let $\mathcal{G}=(V, E)$ be a distance-regular graph with intersection numbers $\left\{p_{i j}^{k}\right\}$. Then $\Gamma(\mathcal{G})$ is invariant under the action of the quantum components $A^{\epsilon}, \epsilon \in$ $\{+,-, \circ\}$, of the adjacency matrix $A$ and

$$
\begin{aligned}
& A^{+} \Phi_{n}=\sqrt{p_{1, n}^{n+1} p_{1, n+1}^{n}} \Phi_{n+1}, \quad n=0,1,2, \ldots, \\
& A^{-} \Phi_{0}=0, \quad A^{-} \Phi_{n}=\sqrt{p_{1, n-1}^{n} p_{1, n}^{n-1}} \Phi_{n-1}, \quad n=1,2, \ldots, \\
& A^{\circ} \Phi_{n}=p_{1, n-1}^{n-1} \Phi_{n}, \quad n=0,1,2, \ldots
\end{aligned}
$$

In particular, $\left(\Gamma(\mathcal{G}), A^{+}, A^{-}\right)$is an interacting Fock space associated with the Jacobi sequence $\left\{p_{1, n-1}^{n} p_{1, n}^{n-1} ; n=1,2, \ldots\right\}$.

The proof is straightforward from definition. In general,

$$
\langle A\rangle=\left\langle\delta_{o}, A \delta_{o}\right\rangle=0, \quad\left\langle A^{2}\right\rangle=\left\langle\delta_{o}, A^{2} \delta_{o}\right\rangle=\kappa(o),
$$

where $\kappa(o)$ is the degree of $o$, i.e., the number of vertices adjacent to $o$. For a distanceregular graph we have $\kappa(o)=p_{11}^{0}$ so that $A / \sqrt{p_{11}^{0}}$ is a proper normalization of the adjacency matrix $A$.

THEOREM 3.2. Let $\left\{\mathcal{G}^{(\nu)}=\left(V^{(\nu)}, E^{(\nu)}\right)\right\}$ be a growing family of distance-regular graphs. Let $A_{\nu}$ and $\left\{p_{i j}^{k}(\nu)\right\}$ be the adjancency matrix and the intersection numbers of $\mathcal{G}_{\nu}$, respectively. Assume that the limits

$$
\omega_{n}=\lim _{\nu} \frac{p_{1, n-1}^{n}(\nu) p_{1, n}^{n-1}(\nu)}{p_{11}^{0}(\nu)}, \quad \alpha_{n}=\lim _{\nu} \frac{p_{1, n-1}^{n-1}(\nu)}{\sqrt{p_{11}^{0}(\nu)}}
$$

exist for all $n=1,2, \ldots$ Let $\Gamma_{\left\{\omega_{n}\right\}}=\left(\Gamma,\left\{\Psi_{n}\right\}, B^{+}, B^{-}\right)$be the interacting Fock space associated with $\left\{\omega_{n}\right\}$ and define a diagonal operator $B^{\circ}$ by $B^{\circ} \Psi_{n}=\alpha_{n+1} \Psi_{n}$. Then for 
the quantum components $A_{\nu}^{\epsilon}, \epsilon \in\{+,-, \circ\}$, of the adjacency matrix $A_{\nu}$,

$$
\lim _{\nu} \frac{A_{\nu}^{\epsilon}}{\sqrt{p_{11}^{0}(\nu)}}=B^{\epsilon}, \quad \epsilon \in\{+,-, \circ\}
$$

in the stochastic sense.

The stochastic convergence (3.2) means that for any choice of a finite number of symbols $\epsilon_{1}, \ldots, \epsilon_{m}, \epsilon_{i} \in\{+,-, \circ\}$, we have

$$
\lim _{\nu}\left\langle\Phi_{0}, \frac{A_{\nu}^{\epsilon_{m}}}{\sqrt{p_{11}^{0}(\nu)}} \ldots \frac{A_{\nu}^{\epsilon_{1}}}{\sqrt{p_{11}^{0}(\nu)}} \Phi_{0}\right\rangle=\left\langle\Psi_{0}, B^{\epsilon_{m}} \ldots B^{\epsilon_{1}} \Psi_{0}\right\rangle .
$$

Theorem 3.2 does not appear explicitly in the literature, however, the proof is essentially a combination of the arguments in Hora-Obata $[17,18]$.

Once we grasp an interacting Fock space structure, the computation of $\mu$ in the main question falls into a classical calculus. Let $\Gamma_{\left\{\omega_{n}\right\}}=\left(\Gamma,\left\{\Psi_{n}\right\}, B^{+}, B^{-}\right)$be the interacting Fock space associated with a Jacobi sequence $\left\{\omega_{n}\right\}$ and $B^{\circ}$ a diagonal operator defined by $B^{\circ} \Psi_{n}=\alpha_{n+1} \Psi_{n}$, where $\left\{\alpha_{n}\right\}$ is a real sequence. It is easily checked with the help of the Hamburger theorem (see e.g., Chihara [7, Chapter II], Shohat-Tamarkin [25, Theorem 1.2]) that there exists a Borel probability measure $\mu$ on $\mathbb{R}$ such that

$$
\left\langle\Psi_{0},\left(B^{+}+B^{-}+B^{\circ}\right)^{m} \Psi_{0}\right\rangle=\int_{-\infty}^{+\infty} x^{m} \mu(d x), \quad m=1,2, \ldots
$$

In general, $\mu$ is not uniquely determined due to the famous determinate moment problem.

Proposition 3.3. If $\omega_{n}=O\left((n \log n)^{2}\right)$ and $\alpha_{n}=O(n \log n)$, there exists a unique Borel probability measure $\mu$ satisfying (3.3).

Proof. By the Accardi-Bożejko formula [2] we know that

$$
M_{m} \equiv\left\langle\Psi_{0},\left(B^{+}+B^{-}+B^{\circ}\right)^{m} \Psi_{0}\right\rangle=\sum_{\vartheta \in \mathcal{P}_{\mathrm{NCPS}}(m)} \prod_{\substack{v \in \vartheta \\|v|=1}} \alpha\left(d_{\vartheta}(v)\right) \prod_{\substack{v \in \vartheta \\|v|=2}} \omega\left(d_{\vartheta}(v)\right),
$$

where $\mathcal{P}_{\mathrm{NCPS}}(m)$ stands for the set of non-crossing pair partitions with singletons of $\{1,2, \ldots, m\}$ and $d_{\vartheta}(v) \geq 1$ the depth of a block $v$ in the partition $\vartheta$. Choose $C>0$ such that

$$
\omega_{n} \leq C^{2}(n \log n+1)^{2}, \quad \alpha_{n} \leq C(n \log n+1), \quad n=1,2, \ldots
$$

Combining the simple estimate $\left|\mathcal{P}_{\mathrm{NCPS}}(m)\right| \leq 3^{m}$, we obtain

$$
M_{m} \leq(3 C)^{m}\left(\frac{m+1}{2} \log \frac{m+1}{2}+1\right)^{m} .
$$

Then the condition of Carleman's moment test (see e.g., Shohat-Tamarkin [25, Theorem 1.10]) is satisfied:

$$
\sum_{m=1}^{\infty} M_{2 m}^{-\frac{1}{2 m}}=+\infty,
$$

so that $\mu$ is uniquely determined by its moment sequence $\left\{M_{m}\right\}$. 
4. The odd graph. Let $k \geq 2$ be a fixed integer and put $\Omega=\{1,2, \ldots, 2 k-1\}$. Let $V$ be the set of subsets $x$ of $\Omega$ having cardinality $k-1$, i.e.,

$$
V=\{x \subset \Omega ;|x|=k-1\},
$$

and put

$$
E=\{\{x, y\} ; x, y \in V, x \cap y=\emptyset\} .
$$

The graph $(V, E)$ is called the odd graph of degree $k$ and is denoted by $O_{k}$. Obviously, $O_{k}$ is a regular graph of degree $k$.

The odd graphs have been studied in algebraic graph theory, as a natural series containing the Petersen graph as $\mathrm{O}_{3}$, and some of their properties are found in Biggs $[3,4]$. For being self-contained we shall derive necessary properties from the following useful description of the distance function. The proof is deferred to Section A.

Proposition 4.1. Let $O_{k}$ be an odd graph of degree $k \geq 2$. For $n=0,1,2, \ldots, k-1$ we define $I_{n}$ by

$$
I_{n}= \begin{cases}k-1-\frac{n}{2}, & \text { if } n \text { is even, } \\ \frac{n-1}{2}, & \text { if } n \text { is odd. }\end{cases}
$$

For a pair of vertices $x, y \in V$ we have

$$
|x \cap y|=I_{n} \quad \Leftrightarrow \quad \partial(x, y)=n .
$$

Corollary 4.2. $\operatorname{diam}\left(O_{k}\right)=k-1$.

Proof. As is easily seen from the definition (4.1), $I$ is a bijection from $\{0,1, \ldots, k-1\}$ onto itself. Then, the maximal distance between two vertices is $k-1$.

COROLlary 4.3. The odd graph $O_{k}$ is distance-transitive, therefore distance-regular.

Proof. Any bijection $\pi: \Omega \rightarrow \Omega$ induces a bijection $\tilde{\pi}: V \rightarrow V$ in a natural manner. Then $\tilde{\pi}$ becomes an automorphism of the graph $O_{k}$, since $|x \cap y|$ is kept invariant under $\tilde{\pi}$. Now let $x, y, x^{\prime}, y^{\prime} \in V$ such that $\partial(x, y)=\partial\left(x^{\prime}, y^{\prime}\right)=n$. By Proposition 4.1 we may set

$$
x=\left\{\alpha_{1}, \ldots, \alpha_{I}, \beta_{1}, \ldots, \beta_{J}\right\}, \quad y=\left\{\alpha_{1}, \ldots, \alpha_{I}, \gamma_{1}, \ldots, \gamma_{J}\right\},
$$

where $I=I_{n}, I+J=k-1,\left\{\beta_{1}, \ldots, \beta_{J}\right\} \cap\left\{\gamma_{1}, \ldots, \gamma_{J}\right\}=\emptyset$. Similarly,

$$
x^{\prime}=\left\{\alpha_{1}^{\prime}, \ldots, \alpha_{I}^{\prime}, \beta_{1}^{\prime}, \ldots, \beta_{J}^{\prime}\right\}, \quad y^{\prime}=\left\{\alpha_{1}^{\prime}, \ldots, \alpha_{I}^{\prime}, \gamma_{1}^{\prime}, \ldots, \gamma_{J}^{\prime}\right\} .
$$

Take a bijection $\pi: \Omega \rightarrow \Omega$ satisfying

$$
\pi\left(\alpha_{i}\right)=\alpha_{i}^{\prime}, \quad \pi\left(\beta_{i}\right)=\beta_{i}^{\prime}, \quad \pi\left(\gamma_{i}\right)=\gamma_{i}^{\prime} .
$$

Then the automorphism $\tilde{\pi}$ satisfies $\tilde{\pi}(x)=x^{\prime}$ and $\tilde{\pi}(y)=y^{\prime}$, which means that $O_{k}$ is distance-transitive.

5. Quantum central limit theorem for odd graphs. Having observed the odd graphs $\left\{O_{k}\right\}$ form a growing family of distance-regular graphs, we shall in this section investigate an asymptotic spectral distribution of the adjacency matrix $A_{k}$ as $k \rightarrow \infty$ by 
applying quantum probabilistic techniques (Theorem 3.2). Our first task is to compute the intersection numbers of $O_{k}$ which are required in Theorem 3.2.

Proposition 5.1. Let $\left\{p_{i j}^{h}\right\}$ be the intersection numbers of the odd graph $O_{k}, k \geq 2$. For $1 \leq n \leq k-1$,

$$
p_{1, n-1}^{n}= \begin{cases}\frac{n}{2}, & \text { if } n \text { is even } \\ \frac{n+1}{2}, & \text { if } n \text { is odd. }\end{cases}
$$

For $0 \leq n \leq k-2$,

$$
p_{1, n+1}^{n}= \begin{cases}k-\frac{n}{2}, & \text { if } n \text { is even }, \\ k-\frac{n+1}{2}, & \text { if } n \text { is odd }\end{cases}
$$

For $0 \leq n \leq k-1$,

$$
p_{1, n}^{n}= \begin{cases}0, & \text { if } 1 \leq n \leq k-2, \\ \frac{k+1}{2}, & \text { if } n=k-1 \text { and } k \text { is odd } \\ \frac{k}{2}, & \text { if } n=k-1 \text { and } k \text { is even. }\end{cases}
$$

Proof. Just a routine application of Proposition 4.1. We shall prove the first identity only. Let $n$ be an even number such that $1 \leq n \leq k-1$. Without loss of generality we set

$$
o=\{1,2, \ldots, k-1\}, \quad x=\left\{1,2, \ldots, I_{n}, k, k+1, \ldots, 2 k-I_{n}-2\right\} .
$$

Then $|o \cap x|=I_{n}$ so that $\partial(o, x)=n$. Let us find a general form of $y$ such that $\partial(x, y)=1$ and $\partial(o, y)=n-1$. In order that $\partial(x, y)=1$ we have by definition

$$
y \subset\left\{I_{n}+1, \ldots, k-1\right\} \cup\left\{2 k-I_{n}-1, \ldots, 2 k-1\right\} .
$$

Since $|y|=k-1$ and $\left|\left\{I_{n}+1, \ldots, k-1\right\} \cup\left\{2 k-I_{n}-1, \ldots, 2 k-1\right\}\right|=k$, the vertex $y$ is obtained by eliminating one element from the right hand side of (5.1). There are two cases: (i) If $y$ is obtained by eliminating one element in $\left\{I_{n}+1, \ldots, k-1\right\}$, we have

$$
|o \cap y|=k-I_{n}-2=\frac{n}{2}-1=\frac{(n-1)-1}{2}=I_{n-1},
$$

where we used (4.1) with $n$ being even. Hence $\partial(o, y)=n-1$. (ii) If $y$ is obtained by eliminating one element in $\left\{2 k-I_{n}-1, \ldots, 2 k-1\right\}$, we have

$$
|o \cap y|=k-I_{n}-1=\frac{n}{2} \neq I_{n-1},
$$

which means that $\partial(o, y) \neq n-1$. Consequently, a vertex $y$ satisfying $\partial(x, y)=1$ and $\partial(o, y)=n-1$ is obtained only in the case (i) and the number of such $y$ 's is

$$
(k-1)-\left(I_{n}+1\right)+1=k-I_{n}-1=\frac{n}{2} .
$$

This proves that $p_{1, n-1}^{n}=n / 2$ for an even $n$.

We are now in a position to state the quantum central limit theorem for the odd graphs $\left\{O_{k}\right\}$. 
THEOREM 5.2. Let $A_{k}$ be the adjacency matrix of the odd graph $O_{k}$ and $A_{k}^{\epsilon}$ its quantum components, $\epsilon \in\{+,-, \circ\}$. Let $\Gamma_{\left\{\omega_{n}\right\}}=\left(\Gamma,\left\{\Psi_{n}\right\}, B^{+}, B^{-}\right)$be the interacting Fock space associated with a Jacobi sequence defined by

$$
\left\{\omega_{n}\right\}_{n=1}^{\infty}=\{1,1,2,2,3,3,4,4, \ldots\} .
$$

Then

$$
\lim _{k \rightarrow \infty} \frac{A_{k}^{ \pm}}{\sqrt{k}}=B^{ \pm}, \quad \lim _{k \rightarrow \infty} \frac{A_{k}^{\circ}}{\sqrt{k}}=0,
$$

in the stochastic sense.

Proof. Let $\left\{p_{i j}^{h}(k)\right\}$ denote the intersection numbers of $O_{k}, k \geq 2$. In view of Theorem 3.2 we only need to find the limits:

$$
\omega_{n}=\lim _{k \rightarrow \infty} \frac{p_{1, n-1}^{n}(k) p_{1, n}^{n-1}(k)}{p_{11}^{0}(k)}, \quad \alpha_{n}=\lim _{k \rightarrow \infty} \frac{p_{1, n-1}^{n-1}(k)}{\sqrt{p_{11}^{0}(k)}},
$$

which are computed with the help of Proposition 5.1. In fact, if $n$ is odd, we have

$$
\omega_{n}=\lim _{k \rightarrow \infty} \frac{1}{k} \cdot \frac{n+1}{2}\left(k-\frac{n-1}{2}\right)=\frac{n+1}{2} .
$$

If $n$ is even,

$$
\omega_{n}=\lim _{k \rightarrow \infty} \frac{1}{k} \cdot \frac{n}{2}\left(k-\frac{n}{2}\right)=\frac{n}{2} .
$$

Thus, $\left\{\omega_{n}\right\}=\{1,1,2,2,3,3, \ldots\}$ as desired. Similarly, we obtain $\left\{\alpha_{n} \equiv 0\right\}$.

We now give an intermediate answer to our main question for the odd graphs. (The complete answer will be given in Theorem 6.1.)

THEOREM 5.3. Let $A_{k}$ be the adjacency matrix of the odd graph $O_{k}, k \geq 2$. Then there exists a unique Borel probability measure $\mu$ on $\mathbb{R}$ such that

$$
\lim _{k \rightarrow \infty}\left\langle\left(\frac{A_{k}}{\sqrt{k}}\right)^{m}\right\rangle=\int_{-\infty}^{+\infty} x^{m} \mu(d x), \quad m=1,2, \ldots
$$

The Jacobi parameter of $\mu$ is given by

$$
\left\{\omega_{n}\right\}_{n=1}^{\infty}=\{1,1,2,2,3,3,4,4, \ldots\}, \quad\left\{\alpha_{n} \equiv 0\right\} .
$$

In particular, $\mu$ is symmetric.

Proof. We maintain the notation in Theorem 5.2. Taking $A_{k}=A_{k}^{+}+A_{k}^{-}+A_{k}^{\circ}$ into account, we see from Theorem 5.2 that

$$
\lim _{k \rightarrow \infty}\left\langle\left(\frac{A_{k}}{\sqrt{k}}\right)^{m}\right\rangle=\left\langle\Psi_{0},\left(B^{+}+B^{-}\right)^{m} \Psi_{0}\right\rangle, \quad m=1,2, \ldots
$$

On the other hand, since $\left\{\omega_{n}\right\}$ and $\left\{\alpha_{n}\right\}$ satisfy the condition in Proposition 3.3, there exists a unique Borel probability measure $\mu$ on $\mathbb{R}$ such that

$$
\left\langle\Psi_{0},\left(B^{+}+B^{-}\right)^{m} \Psi_{0}\right\rangle=\int_{-\infty}^{+\infty} x^{m} \mu(d x), \quad m=1,2, \ldots
$$

Thus, $\mu$ in (5.3) is uniquely determined. That $\mu$ is symmetric follows from $\left\{\alpha_{n} \equiv 0\right\}$ and the uniqueness. 
REMARK 5.4. Since $O_{k}=(V, E)$ is a finite distance-regular graph, the vacuum state coincides with the trace. Thus,

$$
\left\langle\left(\frac{A_{k}}{\sqrt{k}}\right)^{m}\right\rangle=\frac{1}{|V|} \operatorname{Tr}\left[\left(\frac{A_{k}}{\sqrt{k}}\right)^{m}\right]=\frac{(k-1) ! k !}{(2 k-1) ! k^{m / 2}} \operatorname{Tr}\left(A_{k}^{m}\right) .
$$

6. Calculating the limit measure $\mu$. We shall obtain an explicit description of the Borel probability measure $\mu$ in Theorem 5.3, where the Jacobi parameter $\left(\left\{\omega_{n}\right\},\left\{\alpha_{n}\right\}\right)$ is already obtained. Since $\mu$ is uniquely determined by its moment sequence, by general theory (see e.g., Shohat-Tamarkin [25, Chapter II]) the Stieltjes transform of $\mu$ admits a (convergent) continued fraction expansion:

$$
G(z)=\int_{-\infty}^{+\infty} \frac{\mu(d x)}{z-x}=\frac{1}{z}-\frac{1}{z}-\frac{1}{z}-\frac{2}{z}-\frac{2}{z}-\frac{3}{z}-\frac{3}{z}-\frac{4}{z}-\frac{4}{z}-\ldots,
$$

where $z \in\{\operatorname{Im} z \neq 0\}$.

Let us compute the continued fraction (6.1). For $n=1,2, \ldots$ we define a linear fractional transformation:

$$
\sigma_{n}(w)=\frac{n}{z-w} .
$$

Then the $2 n$-th approximant is obtained by

$$
G_{2 n}(z)=\frac{1}{z}-\overbrace{\frac{1}{z}-\frac{1}{z}-\frac{2}{z}-\frac{2}{z}-\cdots-\frac{n}{z}-\frac{n}{z}}^{2 n \text { terms }}=\sigma_{1} \sigma_{1}^{2} \ldots \sigma_{n}^{2}(0) .
$$

On the other hand, using

$$
\sigma_{n}^{2}(w)=\frac{n}{z-\sigma_{n}(w)}=\frac{n}{z}+\frac{n^{2} / z}{z^{2}-n-z w},
$$

we obtain

$$
\sigma_{1}^{2} \ldots \sigma_{n}^{2}(0)=\frac{1}{z}\left\{1+\frac{1^{2}}{z^{2}-3}-\frac{2^{2}}{z^{2}-5}-\frac{3^{2}}{z^{2}-7}-\cdots-\frac{(n-1)^{2}}{z^{2}-(2 n-1)}-\frac{n^{2}}{z^{2}-n}\right\} .
$$

Then (6.2) becomes

$$
G_{2 n}(z)=\frac{z}{z^{2}-1}-\frac{1^{2}}{z^{2}-3}-\frac{2^{2}}{z^{2}-5}-\frac{3^{2}}{z^{2}-7}-\cdots-\frac{(n-1)^{2}}{z^{2}-(2 n-1)}-\frac{n^{2}}{z^{2}-n} .
$$

Since the continued fraction in (6.1) converges, we have

$$
\begin{aligned}
G(z) & =\lim _{n \rightarrow \infty} G_{2 n}(z) \\
& =\frac{z}{z^{2}-1}-\frac{1^{2}}{z^{2}-3}-\frac{2^{2}}{z^{2}-5}-\frac{3^{2}}{z^{2}-7}-\cdots-\frac{(n-1)^{2}}{z^{2}-(2 n-1)}-\cdots,
\end{aligned}
$$

for $z \in\{\operatorname{Im} z \neq 0\}$.

Here we recall the Stieltjes transform of the exponential distribution:

$$
\int_{0}^{+\infty} \frac{e^{-x}}{z-x} d x=\frac{1}{z-1}-\frac{1^{2}}{z-3}-\frac{2^{2}}{z-5}-\frac{3^{2}}{z-7}-\ldots, \quad z \notin[0,+\infty) .
$$

This is verified as a particular case of the continued fraction expansion for the quotient of hypergeometric functions (see Wall [29, Chap. XVIII (92.7)]), or indirectly through the 
Jacobi parameter of the exponential distribution. Comparing (6.4) and (6.5), we obtain

$$
G(z)=\int_{0}^{+\infty} \frac{z e^{-x}}{z^{2}-x} d x .
$$

Then, replacing $x$ with $x^{2}$ and applying partial fraction, we have

$$
G(z)=\int_{0}^{+\infty} \frac{2 x z e^{-x^{2}}}{z^{2}-x^{2}} d x=\int_{0}^{+\infty} \frac{x e^{-x^{2}}}{z-x} d x+\int_{-\infty}^{0} \frac{-x e^{-x^{2}}}{z-x} d x=\int_{-\infty}^{+\infty} \frac{|x| e^{-x^{2}}}{z-x} d x .
$$

Consequently, the probability measure $\mu$ in (6.1) is given by

$$
\mu(d x)=|x| e^{-x^{2}} d x,
$$

which may be called the two-sided Rayleigh distribution following Papoulis [23, p. 78], see Figure 2 for the shape. Using the above explicit form, we may rephrase Theorem 5.3.

ThEOREM 6.1. For the adjacency matrix $A_{k}$ of the odd graph $O_{k}$ we have

$$
\lim _{k \rightarrow \infty}\left\langle\left(\frac{A_{k}}{\sqrt{k}}\right)^{m}\right\rangle=\int_{-\infty}^{+\infty} x^{m}|x| e^{-x^{2}} d x, \quad m=1,2, \ldots
$$

Calculation of the right hand side of (6.7) is elementary.

Proposition 6.2. For the two-sided Rayleigh distribution (6.6) the moments of odd orders vanish and those of even orders are given by

$$
\int_{-\infty}^{+\infty} x^{2 m}|x| e^{-x^{2}} d x=m !, \quad m=0,1,2, \ldots
$$

REMARK 6.3. For the two-sided Rayleigh distribution (6.6) we have obtained explicitly the Jacobi parameter $\left(\left\{\omega_{n}\right\},\left\{\alpha_{n}\right\}\right)$ and the moment sequence $\left\{M_{m}\right\}$. Applying the Accardi-Bożejko formula [2] (see also the proof of Proposition 3.3), we come to the combinatorial identity:

$$
\sum_{\vartheta \in \mathcal{P}_{\mathrm{NCP}}(2 m)} \prod_{v \in \vartheta} \omega\left(d_{\vartheta}(v)\right)=m !, \quad m=1,2, \ldots,
$$

where $\left\{\omega_{n}\right\}=\{1,1,2,2,3,3, \ldots\}$. A direct proof of (6.8) is not known to the authors. If the left hand side of (6.8) were computed in a smart manner, we could avoid continued fractions to obtain the moment sequence. The explicit form of $\mu$ in Theorem 5.3 then follows from the generating function.

REMARK 6.4. The orthogonal polynomials associated with the two-sided Rayleigh distribution are called the generalized Hermite polynomials with parameter $1 / 2$ by Chihara [7, p. 157], see also Szegö [26, p. 380].

7. Spidernet. We start with some notation. Given a graph $\mathcal{G}=(V, E)$ with an origin $o \in V$, consider the stratification as in (2.1). For $x \in V$ we set

$$
\omega_{\epsilon}(x)=|\{y \in V ; y \sim x, \partial(o, y)-\partial(o, x)=\epsilon\}|, \quad \epsilon \in\{+,-, \circ\} .
$$


Let $\kappa(x)$ denote the degree of $x \in V$ as usual. Then we have

$$
\kappa(x)=\omega_{+}(x)+\omega_{-}(x)+\omega_{\circ}(x), \quad x \in V .
$$

Let $a, b, c$ be integers such that $a \geq 1, b \geq 2$ and $1 \leq c \leq b-1$. A spidernet is a graph which satisfies the conditions:

$$
\left\{\begin{array} { l } 
{ \omega _ { + } ( o ) = a , } \\
{ \omega _ { - } ( o ) = 0 , } \\
{ \omega _ { \circ } ( o ) = 0 , }
\end{array} \quad \left\{\begin{array}{l}
\omega_{+}(x)=c, \\
\omega_{-}(x)=1, \\
\omega_{\circ}(x)=b-1-c,
\end{array} \quad \text { for } x \neq o .\right.\right.
$$

Such a spidernet is denoted by $S(a, b, c)$ though not uniquely determined by $(a, b, c)$ in general. Examples are shown in Figure 1. We note that

$$
\kappa(x)= \begin{cases}a, & x=o, \\ b, & x \neq o,\end{cases}
$$

which follows from (7.1) and (7.2). Hence a spidernet is not necessarily a regular graph but may possess high symmetry. Note also that a spidernet $S(a, b, c)$ with $c=b-1$ is a tree. The spidernets have been studied for their interesting spectral geometric properties, see e.g., Urakawa [27], where a spidernet is called a semi-regular graph.
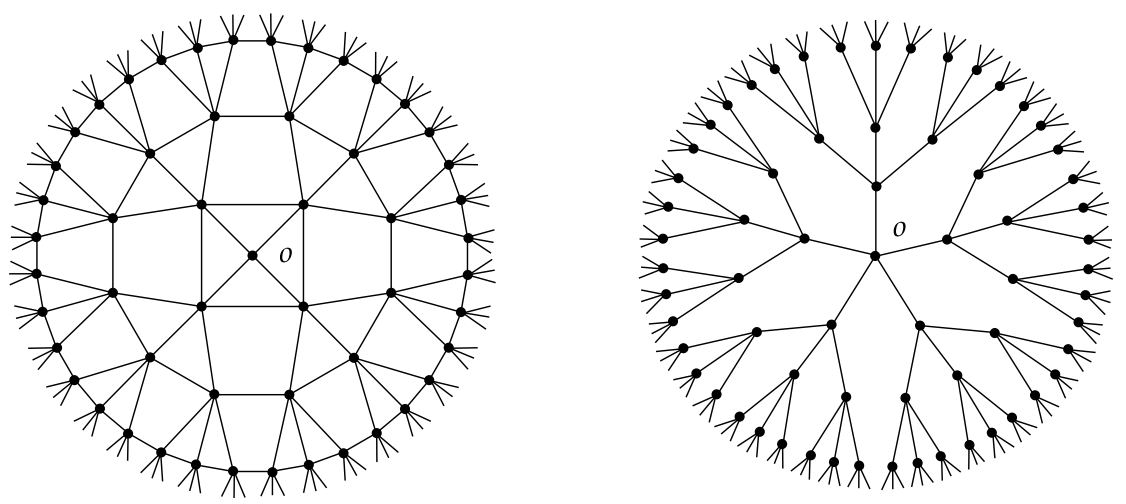

Fig. 1. Spidernets $S(4,6,3)$ and $S(5,4,3)$

Proposition 7.1. Let $A$ be the adjacency matrix of a spidernet $S=S(a, b, c)$. Then $\Gamma(S)$ is invariant under the action of the quantum components $A^{\epsilon}, \epsilon \in\{+,-, \circ\}$. Moreover, we have

$$
\begin{aligned}
& A^{+} \Phi_{0}=\sqrt{a} \Phi_{1}, \quad A^{+} \Phi_{n}=\sqrt{c} \Phi_{n+1}, \quad n=1,2, \ldots, \\
& A^{-} \Phi_{0}=0, \quad A^{-} \Phi_{1}=\sqrt{a} \Phi_{0}, \quad A^{-} \Phi_{n}=\sqrt{c} \Phi_{n-1}, \quad n=2,3, \ldots, \\
& A^{\circ} \Phi_{0}=0, \quad A^{\circ} \Phi_{n}=(b-1-c) \Phi_{n}, \quad n=1,2, \ldots
\end{aligned}
$$

Proof. It is easily derived from (7.2) that

$$
\left|V_{0}\right|=1, \quad\left|V_{n}\right|=a c^{n-1}, \quad n=1,2, \ldots
$$


For $n=0,1,2, \ldots$ we have

$$
\sqrt{\left|V_{n}\right|} A^{+} \Phi_{n}=\sum_{x \in V_{n}} A^{+} \delta_{x}=\sum_{y \in V_{n+1}} \delta_{y}=\sqrt{\left|V_{n+1}\right|} \Phi_{n+1} .
$$

This proves (7.3). The rest is similarly proved.

Proposition 7.1 says that $\left(\Gamma(S),\left\{\Phi_{n}\right\}, A^{+}, A^{-}\right)$is an interacting Fock space with Jacobi parameter

$$
\omega_{1}=a, \quad \omega_{2}=\omega_{3}=\cdots=c
$$

and $A^{\circ}$ is a diagonal operator defined by $A^{\circ} \Phi_{n}=\alpha_{n+1} \Phi_{n}$ with

$$
\alpha_{1}=0, \quad \alpha_{2}=\alpha_{3}=\cdots=b-1-c .
$$

With the help of Proposition 3.3 we see that there exists a unique Borel probability measure $\mu$ on $\mathbb{R}$ such that

$$
\left\langle A^{m}\right\rangle=\left\langle\Phi_{0},\left(A^{+}+A^{-}+A^{\circ}\right)^{m} \Phi_{0}\right\rangle=\int_{-\infty}^{+\infty} x^{m} \mu(d x), \quad m=1,2, \ldots
$$

To be slightly more general, with three real numbers $p>0, q \geq 0, a \in \mathbb{R}$, we may associate a Borel probability measure $\mu_{p, q, a}$ uniquely specified by

$$
\int_{-\infty}^{+\infty} \frac{\mu_{p, q, a}(d x)}{z-x}=\frac{1}{z}-\frac{p}{z-a}-\frac{q}{z-a}-\frac{q}{z-a}-\frac{q}{z-a}-\cdots
$$

In other words, $\mu_{p, q, a}$ is characterized by a Jacobi parameter $(\{p, q, q, \ldots\},\{0, a, a, \ldots\})$. Note that $\mu_{p, q, a}$ has mean zero and variance $p$. We call the probability measure $\mu_{p, q, a}$ the free Meixner law with parameter $(p, q, a)$ after Bożejko-Bryc [5]. The density function is known, see Section B. In particular, the density function of $\mu_{4,3,2}$ shown in Figure 2 is obtained from the spidernet $S(4,6,3)$.

REMARK 7.2. The free Meixner laws of Bożejko-Bryc [5] are parametrized by $a \in \mathbb{R}$ and $b \geq-1$. Their free Meixner law with parameter $(a, b)$ is normalized to have variance one and coincides with $\mu_{1, b+1, a}$ in our definition, see also Bożejko-Wysoczański [6] for a particular subclass of free Meixner laws.

With this notation we claim the following

ThEOREM 7.3. Let $A=A_{a, b, c}$ be the adjacency matrix of a spidernet $S(a, b, c)$. Then

$$
\left\langle A^{m}\right\rangle=\int_{-\infty}^{+\infty} x^{m} \mu_{a, c, b-1-c}(d x), \quad m=1,2, \ldots,
$$

where $\mu_{a, c, b-1-c}$ is the free Meixner law with parameter $(a, c, b-1-c)$.

Proof. The Jacobi parameter of $\mu$ in (7.8) is given by (7.6) and (7.7). Hence $\mu$ is the free Meixner law with parameter $(a, c, b-1-c)$.

REMARK 7.4. For $\kappa \geq 2$ the spidernet $S(\kappa, \kappa, \kappa-1)$ is a homogeneous tree of degree $\kappa$. Let $A_{\kappa}$ be its adjacency matrix. As a direct consequence of Theorem 7.3 we have

$$
\left\langle A_{\kappa}^{m}\right\rangle=\int_{-\infty}^{+\infty} x^{m} \mu_{\kappa, \kappa-1,0}(d x), \quad m=1,2, \ldots
$$


The probability measure $\mu_{\kappa, \kappa-1,0}$ appearing in the right hand side was first obtained by Kesten [21] with a different method. A free Meixner law $\mu_{p, q, 0}$ coincides with the Kesten measure with parameter $(p, q)$, where $p>0$ and $q \geq 0$.

8. Quantum central limit theorem for spidernets. We next consider a growing family of spidernets $S(a, b, c)$ as $a \rightarrow \infty$. Since $\langle A\rangle=0$ and $\left\langle A^{2}\right\rangle=a$, the proper normalization of the adjacency matrix is $A / \sqrt{a}$. In view of Proposition 7.1 we obtain

$$
\begin{array}{ll}
\frac{A^{+}}{\sqrt{a}} \Phi_{0}=\Phi_{1}, & \frac{A^{+}}{\sqrt{a}} \Phi_{n}=\sqrt{\frac{c}{a}} \Phi_{n+1}, \quad n=1,2, \ldots, \\
\frac{A^{-}}{\sqrt{a}} \Phi_{0}=0, & \frac{A^{-}}{\sqrt{a}} \Phi_{1}=\Phi_{0}, \quad \frac{A^{-}}{\sqrt{a}} \Phi_{n}=\sqrt{\frac{c}{a}} \Phi_{n-1}, \quad n=2,3, \ldots, \\
\frac{A^{\circ}}{\sqrt{a}} \Phi_{0}=0, & \frac{A^{\circ}}{\sqrt{a}} \Phi_{n}=\frac{b-1-c}{\sqrt{a}} \Phi_{n}, \quad n=1,2, \ldots
\end{array}
$$

Then, with no difficulty we come to the following

TheOREM 8.1. Let $A=A_{a, b, c}$ be the adjacency matrix of a spidernet $S(a, b, c)$. Let $q \geq 0$ and $r \geq 0$ be real numbers. Let $\left(\Gamma,\left\{\Psi_{n}\right\}, B^{+}, B^{-}\right)$be an interacting Fock space with Jacobi sequence

$$
\omega_{1}=1, \quad \omega_{2}=\omega_{3}=\cdots=q,
$$

and $B^{\circ}$ a diagonal operator defined by $B^{\circ} \Psi_{n}=\alpha_{n+1} \Psi_{n}$ with

$$
\alpha_{1}=0, \quad \alpha_{2}=\alpha_{3}=\cdots=r .
$$

Then in the limit as $a \rightarrow \infty$ with

$$
\frac{c}{a} \rightarrow q, \quad \frac{b-c}{\sqrt{a}} \rightarrow r
$$

we have

$$
\lim \frac{A_{a, b, c}^{\epsilon}}{\sqrt{a}}=B^{\epsilon}, \quad \epsilon \in\{+,-, \circ\},
$$

in the stochastic sense.

The next statement is an answer to our main question for the spidernets.

Theorem 8.2. Let $A=A_{a, b, c}$ be the adjacency matrix of a spidernet $S(a, b, c)$. Let $q \geq 0$ and $r \geq 0$ be real numbers. Then in the limit as $a \rightarrow \infty$ with (8.1) we have

$$
\lim \left\langle\left(\frac{A_{a, b, c}}{\sqrt{a}}\right)^{m}\right\rangle=\int_{-\infty}^{+\infty} x^{m} \mu_{1, q, r}(d x), \quad m=1,2, \ldots,
$$

where $\mu_{1, q, r}$ is the free Meixner law with parameter $(1, q, r)$.

REMARK 8.3. This is a continuation of Remark 7.4. Consider a growing family of homogeneous trees $\{S(\kappa, \kappa, \kappa-1)\}$ as $\kappa \rightarrow \infty$. It then follows directly from Theorem 8.2 that

$$
\lim _{\kappa \rightarrow \infty}\left\langle\left(\frac{A_{\kappa}}{\sqrt{\kappa}}\right)^{m}\right\rangle=\int_{-\infty}^{+\infty} x^{m} \mu_{1,1,0}(d x), \quad m=1,2, \ldots
$$


As is well known, the probability measure $\mu_{1,1,0}$ is the Wigner semicircle law:

$$
\mu_{1,1,0}(d x)=\rho(x) d x, \quad \rho(x)= \begin{cases}\frac{1}{2 \pi} \sqrt{4-x^{2}}, & |x| \leq 2 \\ 0, & \text { otherwise }\end{cases}
$$

In fact, (8.2) is viewed as a prototype of the free central limit theorem of Voiculescu (see [28] and references cited therein) because the adjacency matrix is decomposed into a sum of free independent random variables. Use of various concepts of independence in quantum probability theory is a new promising direction in asymptotic spectral analysis, see Accardi-Ben Ghorbal-Obata [1], Obata [22].

9. Approximation by trees. The argument in Section 7 suggests a direction of generalizing the free Meixner laws from the viewpoint of spectral analysis on graphs.

THEOREM 9.1. Let $\mu$ be a Borel probability measure on $\mathbb{R}$ and $\left(\left\{\omega_{n}\right\},\left\{\alpha_{n}\right\}\right)$ its Jacobi parameter. Assume that $\omega_{n}$ is an integer for all $n$ and $\alpha_{n} \equiv 0$. Then there exists a tree $\mathcal{G}=(V, E)$ with an origin $o \in V$ such that

$$
\left\langle A^{m}\right\rangle=\int_{-\infty}^{+\infty} x^{m} \mu(d x), \quad m=1,2, \ldots
$$

Proof. We shall construct a tree having the desired property. Let $V_{0}, V_{1}, V_{2}, \ldots$ be disjoint sets such that

$$
\left|V_{0}\right|=1, \quad\left|V_{n}\right|=\omega_{n} \ldots \omega_{2} \omega_{1}, \quad n=1,2, \ldots
$$

When $\omega_{n}>0$ for all $n$, we obtain an infinite sequence of non-empty sets. When $\omega_{n}=0$ occurs for some $n$ (then, by definition $\omega_{n+1}=0$ ), we just obtain a finite sequence of non-empty sets. In any case we set

$$
V=\bigcup_{n=0}^{\infty} V_{n}
$$

which is the set of vertices. We introduce a graph structure in $V$ as follows. First the vertex $o \in V_{0}$ is combined with each vertex in $V_{1}$ by an edge. Suppose $n \geq 1$ and edges in $V_{0} \cup V_{1} \cup \cdots \cup V_{n}$ are defined. If $V_{n+1}=\emptyset$, no more edges are defined. We assume that $V_{n+1} \neq \emptyset$. Since $\left|V_{n+1}\right|=\omega_{n+1}\left|V_{n}\right|$, the set $V_{n+1}$ is partitioned into a $\left|V_{n}\right|$ disjoint subsets consisting of $\omega_{n+1}$ vertices:

$$
V_{n+1}=\bigcup_{i=1}^{\left|V_{n}\right|} S_{i}, \quad\left|S_{i}\right|=\omega_{n+1} .
$$

We connect each vertex in $S_{i}$ with the $i$-th vertex in $V_{n}$ by an edge. The graph $\mathcal{G}$ obtained in this way is a tree of which the stratification is given by (9.3). The action of $A$ on $\Gamma(\mathcal{G})$ is easily obtained: First we note that

$$
A \Phi_{0}=\sum_{x \in V_{1}} \delta_{x}=\sqrt{\omega_{1}} \Phi_{1} .
$$


For $n=1,2, \ldots$ we have

$$
\begin{aligned}
\sqrt{\left|V_{n}\right|} A \Phi_{n} & =\sum_{x \in V_{n}} A \delta_{x}=\sum_{y \in V_{n+1}} \delta_{y}+\sum_{z \in V_{n-1}} \omega_{n} \delta_{z} \\
& =\sqrt{\left|V_{n+1}\right|} \Phi_{n+1}+\omega_{n} \sqrt{\left|V_{n-1}\right|} \Phi_{n-1} .
\end{aligned}
$$

Hence in view of (9.2) we come to

$$
A \Phi_{n}=\sqrt{\omega_{n+1}} \Phi_{n+1}+\sqrt{\omega_{n}} \Phi_{n-1}, \quad n=1,2, \ldots
$$

We then see from (9.4) and (9.5) that

$$
\begin{aligned}
& A^{+} \Phi_{n}=\sqrt{\omega_{n+1}} \Phi_{n+1}, \quad n=0,1,2, \ldots, \\
& A^{-} \Phi_{0}=0, \quad A^{-} \Phi_{n}=\sqrt{\omega_{n}} \Phi_{n-1}, \quad n=1,2, \ldots
\end{aligned}
$$

Namely, $\left(\Gamma(\mathcal{G}), A^{+}, A^{-}\right)$is an interacting Fock space with Jacobi parameter $\left\{\omega_{n}\right\}$ and hence (9.1) holds.

Theorem 9.1 suggests that from the viewpoint of spectral analysis a large graph with few edges lying in a stratum is approximated by trees. For example, a large odd graph $O_{k}$ is approximated by a tree with $\left\{\omega_{n}\right\}=\{k, k, 2 k, 2 k, 3 k, 3 k, \ldots\}$ being the parameter in Theorem 9.1. We expect that a spidernet plays a similar role for a more general $\mu$ with non-vanishing $\left\{\alpha_{n}\right\}$.

A. Proof of Proposition 4.1. As is easily verified from the definition (4.1), we have $\left\{I_{0}, I_{1}, \ldots, I_{k-1}\right\}=\{0,1, \ldots, k-1\}$. In other words, regarded as a map $I$ is a bijection from $\{0,1, \ldots, k-1\}$ onto itself. We set

$$
\begin{aligned}
& E_{n}=\left\{(x, y) \in V \times V ;|x \cap y|=I_{n}\right\}, \\
& F_{n}=\{(x, y) \in V \times V ; \partial(x, y)=n\} .
\end{aligned}
$$

It is sufficient to prove that $E_{n}=F_{n}$ for all $n$. For $n=0$ we have $I_{0}=k-1$ so that

$$
\begin{aligned}
E_{0} & =\{(x, y) \in V \times V ;|x \cap y|=k-1\} \\
& =\{(x, y) \in V \times V ; x=y\} \\
& =\{(x, y) \in V \times V ; \partial(x, y)=0\}=F_{0} .
\end{aligned}
$$

For $n=1$ we have $I_{1}=0$ so that

$$
\begin{aligned}
E_{1} & =\{(x, y) \in V \times V ;|x \cap y|=0\} \\
& =\{(x, y) \in V \times V ; x \sim y\} \\
& =\{(x, y) \in V \times V ; \partial(x, y)=1\}=F_{1} .
\end{aligned}
$$

Thus the assertion is true for $n=0,1$. Assuming that the assertion is true up to $n$, $1 \leq n \leq k-2$, we only need to prove that $E_{n+1}=F_{n+1}$.

We shall first prove that $E_{n+1} \subset F_{n+1}$. Take $(x, y) \in E_{n+1}$, which satisfies $|x \cap y|=I_{n+1}$. For simplicity we put $I=I_{n+1}$. Note that $I \neq I_{0}, I_{1}$, i.e., $1 \leq I \leq k-2$. We set

$$
\begin{gathered}
x=\left\{\alpha_{1}, \ldots, \alpha_{I}, \beta_{1}, \ldots, \beta_{J}\right\}, \quad y=\left\{\alpha_{1}, \ldots, \alpha_{I}, \gamma_{1}, \ldots, \gamma_{J}\right\}, \\
\left\{\beta_{1}, \ldots, \beta_{J}\right\} \cap\left\{\gamma_{1}, \ldots, \gamma_{J}\right\}=\emptyset,
\end{gathered}
$$


where $I+J=k-1, I \geq 1$ and $J \geq 1$. In view of $|\Omega-(x \cup y)|=(2 k-1)-(I+2 J)=I+1$ we set

$$
\Omega-(x \cup y)=\left\{\omega_{1}, \ldots, \omega_{I+1}\right\} .
$$

Suppose first that $n$ is even. Then $I=I_{n+1}=n / 2$. Consider

$$
z=\left\{\omega_{1}, \ldots, \omega_{I}, \beta_{1}, \ldots, \beta_{J}\right\} .
$$

Since $z \cap y=\emptyset$ we have $\partial(z, y)=1$. On the other hand, $|x \cap z|=J=k-1-n / 2=I_{n}$ so that $\partial(x, z)=n$ by assumption of induction. Hence

$$
\partial(x, y) \leq \partial(x, z)+\partial(z, y)=n+1 \text {. }
$$

Suppose next that $n$ is odd. Then $I=I_{n+1}=k-1-(n+1) / 2$ and $J=(n+1) / 2$. Consider

$$
w=\left\{\omega_{1}, \ldots, \omega_{I}, \omega_{I+1}, \beta_{1}, \ldots, \beta_{J-1}\right\} .
$$

Obviously, $w \cap y=\emptyset$ and hence $\partial(w, y)=1$. On the other hand, since $|x \cap w|=J-1=$ $(n-1) / 2=I_{n}$, we have $\partial(x, z)=n$ by assumption of induction and

$$
\partial(x, y) \leq \partial(x, w)+\partial(w, y)=n+1 \text {. }
$$

Combining (A.1) and (A.2), we come to $\partial(x, y) \leq n+1$ independent of the parity of $n$. However, $\partial(x, y) \leq n$ does not happen. In fact, if it happens, by assumption of induction we have $(x, y) \in F_{\partial(x, y)}=E_{\partial(x, y)}$ which contradicts $(x, y) \in E_{n+1}$. Consequently, $\partial(x, y)=n+1$ and $E_{n+1} \subset F_{n+1}$.

We prove the converse inclusion. Let $(x, y) \in F_{n+1}$, i.e., $\partial(x, y)=n+1$. Then there exists $z \in V$ such that $\partial(x, z)=n$ and $\partial(z, y)=1$. By assumption of induction we may set

$$
\begin{gathered}
x=\left\{\alpha_{1}, \ldots, \alpha_{I^{\prime}}, \beta_{1}, \ldots, \beta_{J^{\prime}}\right\}, \quad z=\left\{\alpha_{1}, \ldots, \alpha_{I^{\prime}}, \gamma_{1}, \ldots, \gamma_{J^{\prime}}\right\}, \\
\left\{\beta_{1}, \ldots, \beta_{J^{\prime}}\right\} \cap\left\{\gamma_{1}, \ldots, \gamma_{J^{\prime}}\right\}=\emptyset,
\end{gathered}
$$

where $I^{\prime}=I_{n}, I^{\prime}+J^{\prime}=k-1, I^{\prime} \geq 0$ and $J^{\prime} \geq 1$. Set

$$
\Omega-z=\left\{\beta_{1}, \ldots, \beta_{J^{\prime}}, \omega_{1}, \ldots, \omega_{I^{\prime}+1}\right\} .
$$

Since $y$ is adjacent to $z$, it is obtained from $\Omega-z$ by eliminating one element. Namely, $y$ is one of the following types:

$$
\begin{aligned}
& y_{1}=\left\{\beta_{1}, \ldots, \beta_{J^{\prime}}, \omega_{1}, \ldots, \omega_{I^{\prime}+1}\right\}-\left\{\beta_{i}\right\}, \\
& y_{2}=\left\{\beta_{1}, \ldots, \beta_{J^{\prime}}, \omega_{1}, \ldots, \omega_{I^{\prime}+1}\right\}-\left\{\omega_{i}\right\} .
\end{aligned}
$$

As for $y_{1}$, by simple calculation based on definition we have

$$
\left|x \cap y_{1}\right|=J^{\prime}-1=k-2-I_{n}= \begin{cases}I_{n-1} & \text { if } n \text { is even } \\ I_{n+1} & \text { if } n \text { is odd }\end{cases}
$$

Similarly,

$$
\left|x \cap y_{2}\right|=J^{\prime}=k-1-I_{n}= \begin{cases}I_{n+1} & \text { if } n \text { is even } \\ I_{n-1} & \text { if } n \text { is odd }\end{cases}
$$


In any case $|x \cap y|=I_{n \pm 1}$. But by assumption of induction we see that $|x \cap y|=I_{n-1}$ is equivalent to $\partial(x, y)=n-1$, which contradicts $\partial(x, y)=n+1$. Hence $|x \cap y|=I_{n+1}$, which implies that $F_{n+1} \subset E_{n+1}$.

Proposition A.1. The distance on the odd graph $O_{k}$ is given by

$$
\partial(x, y)=\min \{2(k-1-|x \cap y|), 2|x \cap y|+1\}, \quad x, y \in V .
$$

In other words,

$$
\partial(x, y)= \begin{cases}2|x \cap y|+1, & \text { if } \quad 0 \leq|x \cap y| \leq \frac{k-2}{2}, \\ 2(k-1-|x \cap y|), & \text { if } \frac{k-1}{2} \leq|x \cap y| \leq k-1 .\end{cases}
$$

Proof. We regard $I_{n}$ defined in (4.1) as a bijective map from $\{0,1, \ldots, k-1\}$ onto itself. To find the inverse function, we consider the equation $i=I_{n}$. By definition we obtain

$$
n= \begin{cases}2(k-1-i), & \text { if } n \text { is even, } \\ 2 i+1, & \text { if } n \text { is odd, }\end{cases}
$$

By an elementary observation we see that $2(k-1-i) \leq k-1<k \leq 2 i+1$ or $2 i+1 \leq$ $k-1<k \leq 2(k-1-i)$ happens. In order that $0 \leq n \leq k-1$ is fulfilled, we need to choose the smaller one in (A.5). Thus, the inverse map of $I$ is given by

$$
I^{-1}(i)=\min \{2(k-1-i), 2 i+1\} \text {. }
$$

Since $|x \cap y|=I_{\partial(x, y)}$ by Proposition 4.1, applying the inverse map (A.6) we obtain (A.3).

B. Density functions of the free meixner laws. The density function of the free Meixner law $\mu_{p, q, a}(p>0, q \geq 0, a \in \mathbb{R})$ was computed in Cohen-Trenholme [8] and Saitoh-Yoshida [24]. Recall that their parametrization is different from ours (and their papers contain small misprints).

The free Meixner law $\mu_{p, q, a}$ is uniquely specified by its Stieltjes transform given by the convergent continued fraction:

$$
G(z)=\frac{1}{z}-\frac{p}{z-a}-\frac{q}{z-a}-\frac{q}{z-a}-\frac{q}{z-a}-\ldots, \quad \operatorname{Im} z \neq 0 .
$$

The right hand side is easily computed:

$$
G(z)=\frac{(2 q-p) z+p a-p \sqrt{(z-a)^{2}-4 q}}{2(q-p) z^{2}+2 p a z+2 p^{2}},
$$

where the analytic square root is a holomorphic function on $\mathbb{C}-[a-2 \sqrt{q}, a+2 \sqrt{q}]$ and the branch is chosen in such a way that $\sqrt{(z-a)^{2}-4 q}>0$ for $z \in \mathbb{R}$ with $z>a+2 \sqrt{q}$. The absolutely continuous part of $\mu_{p, q, a}(d x)$, denoted by $\rho_{p, q, a}(x)$, is obtained by the Stieltjes inversion formula:

$$
\rho_{p, q, a}(x)=\frac{p}{2 \pi} \frac{\sqrt{4 q-(x-a)^{2}}}{(q-p) x^{2}+p a x+p^{2}}, \quad|x-a| \leq 2 \sqrt{q} .
$$

We note that $\rho_{p, q,-a}(x)=\rho_{p, q, a}(-x)$. For simplicity, we set

$$
g(z)=(q-p) z^{2}+p a z+p^{2}
$$


which appears in the denominator of (B.2). Note that the possible real zeroes of $g(z)$ lies outside the open interval $(-2 \sqrt{q},+2 \sqrt{q})$. We consider the following five cases.

(Case 1) $q=p>0$ and $a=0$. Then the density function becomes

$$
\rho_{p, p, 0}(x)=\frac{1}{2 \pi p} \sqrt{4 p-x^{2}}, \quad|x| \leq 2 \sqrt{p},
$$

which is the Wigner semicircle law with variance $p$. There is no atom and

$$
\mu_{p, p, 0}(d x)=\rho_{p, p, 0}(x) d x .
$$

(Case 2) $q=p>0$ and $a \neq 0$. The density function becomes

$$
\rho_{p, p, a}(x)=\frac{1}{2 \pi} \frac{\sqrt{4 p-(x-a)^{2}}}{a x+p}, \quad|x-a| \leq 2 \sqrt{p} .
$$

Moreover, $\mu_{p, p, a}$ may possess one atom at the zero of $g(z)$, i.e., at $x=\lambda_{0}=-p / a$. The weight is obtained from the residue of $G(z)$. For example, if $a>0$, noting the signature of $\sqrt{(z-a)^{2}-4 q}$ for $z=\lambda_{0} \leq a-2 \sqrt{p}$, we have

$$
\lim _{z \rightarrow \lambda_{0}}\left(z-\lambda_{0}\right) G(z)=\frac{1}{2 a}\left(a-\frac{p}{a}+\left|a-\frac{p}{a}\right|\right),
$$

which is $1-p / a^{2}$ for $a^{2}>p$, and 0 for $a^{2} \leq p$. After similar computation for $a<0$ we obtain

$$
\mu_{p, p, a}(d x)= \begin{cases}\rho_{p, p, a}(x) d x & \text { for } a^{2} \leq p \\ \rho_{p, p, a}(x) d x+\left(1-\frac{p}{a^{2}}\right) \delta_{-p / a} & \text { for } a^{2}>p\end{cases}
$$

In fact, the above result covers (Case 1). We see that $\mu_{p, p, a}$ is an affine transformation of the free Poisson law with parameter $p / a^{2}$ (see e.g., Hiai-Petz [12, Section 3.3]).

We now come to the case where $q \neq p$, that is $g(z)$ is a quadratic function. Set

$$
D=a^{2}-4(q-p)
$$

and consider the following three cases according to the signature of $D$.

(Case 3) $D<0$, that is, $0 \leq a^{2}<4(q-p)$. Then $g(z)$ has no real zeroes so that

$$
\mu_{p, q, a}(d x)=\rho_{p, q, a}(x) d x .
$$

(Case 4) $D=0$, that is, $0<a^{2}=4(q-p)$. Then $g(z)$ has a real multiple zero outside $[a-2 \sqrt{q}, a+2 \sqrt{q}]$, nevertheless $\mu_{p, q, a}$ has no atom and (B.3) holds.

(Case 5) $D>0$, that is, $4(q-p)<a^{2}$. Then $g(z)$ has two real zeroes:

$$
\lambda_{ \pm}=\frac{p}{2(q-p)}(-a \pm \sqrt{D})
$$

and $\mu_{p, q, a}$ is of the form:

$$
\mu_{p, q, a}(d x)=\rho_{p, q, a}(x) d x+w_{+} \delta_{\lambda_{+}}+w_{-} \delta_{\lambda_{-}} .
$$

To describe $w_{ \pm}$we define

$$
\nu_{+}=\frac{1}{\sqrt{D}}\left(\frac{q \lambda_{+}}{p}-\frac{p}{\lambda_{+}}\right), \quad \nu_{-}=\frac{1}{\sqrt{D}}\left(\frac{q \lambda_{-}}{p}-\frac{p}{\lambda_{-}}\right) .
$$


(Case 5-1) $0<4(q-p)<a^{2}$. Then $\lambda_{-}<\lambda_{+}$and both lie in the same half line $(-\infty, a-2 \sqrt{q}]$ or $[a+2 \sqrt{q},+\infty)$. The weights are given as follows:

$$
\begin{aligned}
& w_{+}= \begin{cases}0, & a \leq-2 \sqrt{q-p}, \quad 2 \sqrt{q-p}<a \leq(2 q-p) / \sqrt{q}, \\
\nu_{+}, & a \geq(2 q-p) / \sqrt{q},\end{cases} \\
& w_{-}= \begin{cases}-\nu_{-}, & a \leq-(2 q-p) / \sqrt{q}, \\
0, & -(2 q-p) / \sqrt{q} \leq a<-2 \sqrt{q-p}, \quad 2 \sqrt{q-p}<a .\end{cases}
\end{aligned}
$$

(Case 5-2) $0 \leq q<2 q<p$. Note that $\lambda_{+}<a-2 \sqrt{p}$ and $\lambda_{-}>a+2 \sqrt{p}$. The weights $w_{ \pm}$are given as follows:

$$
w_{+}=\left\{\begin{array}{ll}
0, & a \leq-(p-2 q) / \sqrt{q}, \\
\nu_{+}, & a \geq-(p-2 q) / \sqrt{q},
\end{array} \quad w_{-}= \begin{cases}-\nu_{-}, & a \leq(p-2 q) / \sqrt{q}, \\
0, & a \geq(p-2 q) / \sqrt{q} .\end{cases}\right.
$$

(Case 5-3) $0 \leq q<p<2 q$. The situation is similar to (Case 5-2) and the weights are given as follows:

$$
w_{+}=\left\{\begin{array}{ll}
0, & a \leq(2 q-p) / \sqrt{q}, \\
\nu_{+}, & a \geq(2 q-p) / \sqrt{q},
\end{array} \quad w_{-}= \begin{cases}-\nu_{-}, & a \leq-(2 q-p) / \sqrt{q}, \\
0, & a \geq-(2 q-p) / \sqrt{q}\end{cases}\right.
$$

In fact, (Case 5-2) and (Case 5-3) can be unified:

$$
w_{+}=\frac{1}{2}\left(\left|\nu_{+}\right|+\nu_{+}\right), \quad w_{-}=\frac{1}{2}\left(\left|\nu_{-}\right|-\nu_{-}\right) .
$$
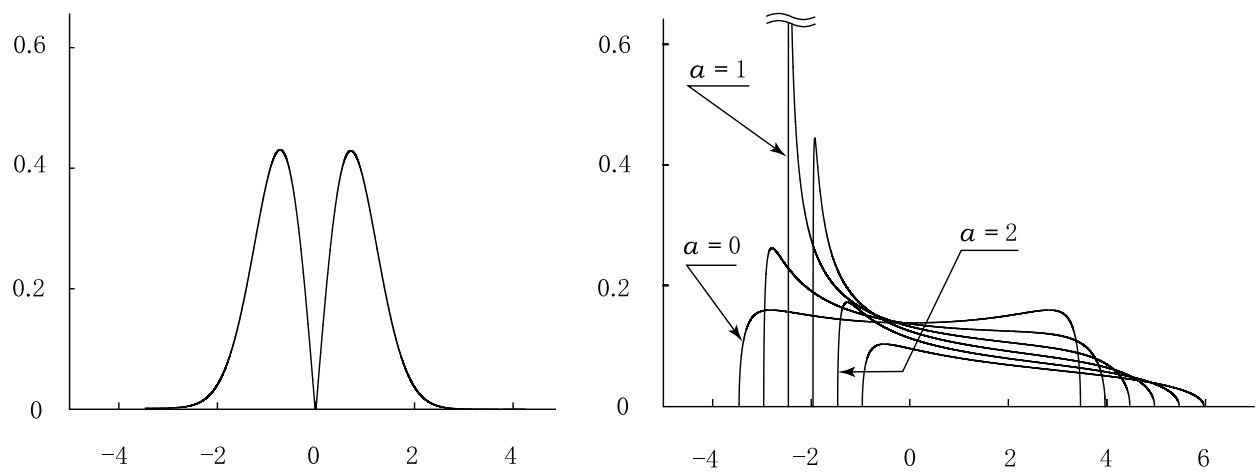

Fig. 2. Two-sided Rayleigh distribution and free Meixner distribution $\mu_{4,3, a}$

Note added in proof. Another Carleman's moment test [25, Sect. 2.17] verifies that Proposition 3.3 remains valid without assuming any condition on $\left\{\alpha_{n}\right\}$.

\section{References}

[1] L. Accardi, A. Ben Ghorbal and N. Obata, Monotone independence, comb graphs and Bose-Einstein condensation, Infin. Dimens. Anal. Quantum Probab. Relat. Top. 7 (2004), 419-435. 
[2] L. Accardi and M. Bożejko, Interacting Fock spaces and Gaussianization of probability measures, Infin. Dimens. Anal. Quantum Probab. Relat. Top. 1 (1998), 663-670.

[3] N. Biggs, Some odd graph theory, Ann. New York Acad. Sci. 319 (1979), 71-81.

[4] N. Biggs, Algebraic Graph Theory, 2nd ed., Cambridge University Press, 1993.

[5] M. Bożejko and W. Bryc, On a class of free Lévy laws related to a regression problem, preprint, 2004, arXiv:math.OA/0410601

[6] M. Bożejko and J. Wysoczański, Remarks on t-transformations of measures and convolutions, Ann. Inst. H. Poincaré Probab. Stat. 37 (2001), 737-761.

[7] T. S. Chihara, An Introduction to Orthogonal Polynomials, Gordon and Breach, 1978.

[8] J. M. Cohen and A. R. Trenholme, Orthogonal polynomials with a constant recursion formula and an application to Harmonic analysis, J. Funct. Anal. 59 (1984), 175-184.

[9] Y. Hashimoto, Quantum decomposition in discrete groups and interacting Fock spaces, Infin. Dimens. Anal. Quantum Probab. Relat. Top. 4 (2001), 277-287.

[10] Y. Hashimoto, A. Hora and N. Obata, Central limit theorems for large graphs: Method of quantum decomposition, J. Math. Phys. 44 (2003), 71-88.

[11] Y. Hashimoto, N. Obata and N. Tabei, A quantum aspect of asymptotic spectral analysis of large Hamming graphs, in: Quantum Information III, T. Hida and K. Saitô (eds.), World Scientific, 2001, 45-57.

[12] F. Hiai and D. Petz, The Semicircle Law, Free Random Variables and Entropy, Amer. Math. Soc., 2000.

[13] A. Hora, Central limit theorems and asymptotic spectral analysis on large graphs, Infin. Dimens. Anal. Quantum Probab. Relat. Top. 1 (1998), 221-246.

[14] A. Hora, Gibbs state on a distance-regular graph and its application to a scaling limit of the spectral distributions of discrete Laplacians, Probab. Theory Relat. Fields 118 (2000), $115-130$.

[15] A. Hora, Scaling limit for Gibbs states for Johnson graphs and resulting Meixner classes, Infin. Dimens. Anal. Quantum Probab. Relat. Top. 6 (2003), 139-143.

[16] A. Hora, Asymptotic spectral analysis on the Johnson graphs in infinite degree and zero temperature limit, Interdiscip. Inform. Sci. 10 (2004), 1-10.

[17] A. Hora and N. Obata, Quantum decomposition and quantum central limit theorem, in: Fundamental Problems in Quantum Physics, L. Accardi and S. Tasaki (eds.), World Scientific, 2003, 284-305.

[18] A. Hora and N. Obata, An interacting Fock space with periodic Jacobi parameter obtained from regular graphs in large scale limit, in: Quantum Information V, T. Hida and K. Saitô (eds.), World Scientific, 2006, 121-144.

[19] A. Hora and N. Obata, Quantum Probability and Spectral Analysis on Graphs, a monograph to appear.

[20] T. Huang and C.-R. Liu, Spectral characterization of some generalized odd graphs, Graphs Combin. 15 (1999), 195-209.

[21] H. Kesten, Symmetric random walks on groups, Trans. Amer. Math. Soc. 92 (1959), 336-354.

[22] N. Obata, Quantum probabilistic approach to spectral analysis of star graphs, Interdiscip. Inform. Sci. 10 (2004), 41-52.

[23] A. Papoulis, Probability, Random Variables, and Stochastic Processes, 2nd ed., McGrawHill, 1984.

[24] N. Saitoh and H. Yoshida, The infinite divisibility and orthogonal polynomials with a constant recursion formula in free probability theory, Probab. Math. Statist. 21 (2001), 159-170. 
[25] J. A. Shohat and J. D. Tamarkin, The Problem of Moments, Amer. Math. Soc., 1943.

[26] G. Szegö, Orthogonal Polynomials, 3rd ed., Amer. Math. Soc., 1967.

[27] H. Urakawa, The Cheeger constant, the heat kernel, and the Green kernel of an infinite graph, Monatsh. Math. 138 (2003), 225-237.

[28] D. Voiculescu, K. Dykema and A. Nica, Free Random Variables, CRM Monograph Series, Amer. Math. Soc., 1992.

[29] H. S. Wall, Analytic Theory of Continued Fraction, AMS Chelsea Pub., 1948. 
